\title{
Volatile Constituents of Five Baccharis Species from Northeastern Argentina
}

\author{
Daiana Retta, ${ }^{*, a}$ Martha Gattuso, ${ }^{b}$ Susana Gattuso, ${ }^{b}$ Paola Di Leo Lira, ${ }^{a}$ \\ Catalina van Baren ${ }^{a}$ and Arnaldo Bandoni ${ }^{a}$
}

\author{
${ }^{a}$ Cátedra de Farmacognosia, Facultad de Farmacia y Bioquímica, Universidad de Buenos Aires, \\ Junín 956, $2^{\circ}$ piso (C 1113 AAD) Buenos Aires, Argentina \\ ${ }^{b}$ Cátedra de Botánica, Facultad de Ciencias Bioquímicas y Farmacéuticas, Universidad Nacional de Rosario, \\ Suipacha 531 (S 2002 LRK) Rosario, Santa Fe, Argentina
}

\begin{abstract}
Os óleos essenciais obtidos por hidrodestilação das partes aéreas de Baccharis gaudichaudiana, B. microcephala, B. penningtonii, B. phyteumoides e B. spicata coletadas em diferentes regiões da Argentina foram analisados qualitativa e quantitativamente utilizando um sistema CG e CG/EM com uma configuração especial. Os rendimentos dos óleos variaram de 0,1 a $0,2 \%$ (m/v de planta seca). Diferentes compostos foram observados na composição dos óleos essenciais das espécies estudadas: $B$. gaudichaudiana apresenta espatulenol e $\beta$-pineno como constituintes principais e, B. microcephala e B. spicata, espatulenol e óxido de cariofileno em concentrações mais elevadas. $\mathrm{Na}$ última espécie citada, o $\beta$-pineno também foi encontrado em quantidades significativas. Germacreno D foi o principal componente presente na $B$. penningtonii. Finalmente, $\beta$-selineno e acetato de fitol $\mathrm{E}$ foram os principais componentes encontrados na $B$. phyteumoides.
\end{abstract}

Essential oils obtained by hydrodistillation from the aerial parts of Baccharis gaudichaudiana, B. microcephala, B. penningtonii, B. phyteumoides and B. spicata collected from different locations in Argentina were analyzed. Qualitative and quantitative analysis were performed using a GC/FID/MS system with a special configuration. Essential oil yields ranged from 0.1 to $0.2 \%$ (m/v of dry material). Different chemical patterns were observed in the essential oils composition of the species studied: spathulenol and $\beta$-pinene were the main constituents in B. gaudichaudiana, whereas caryophyllene oxide and spathulenol were the major constituents in B. microcephala and B. spicata. In this latter species, $\beta$-pinene has also been found in high amounts. Germacrene D was the major compound in B. penningtonii. Finally, $\beta$-selinene and phytol acetate $E$ were the main components in $B$. phyteumoides.

Keywords: Baccharis gaudichaudiana, B. microcephala, B. penningtonii, B. phyteumoides, B. spicata, carqueja, essential oils

\section{Introduction}

The genus Baccharis belongs to the Asteraceae family. This genus comprises more than 400 species in America, mainly South America. ${ }^{1}$ Essential oils from 38 species of this genus have previously been analyzed, accounting in many cases for the value of these plants in traditional medicine as diuretics and stomachics and in fragrance industry. Because of their bitter properties these oils are also frequently used to prepare alcoholic and non-alcoholic beverages. Ninety six species of Baccharis were found in Argentina, and they were classified into 15 sections. Section

*e-mail: dretta@ffyb.uba.ar
Caulopterae or Alatae comprises 10 species, all having stems with 2 or 3 longitudinal lateral wings., ${ }^{2,3}$ The most common Baccharis species used in traditional medicine and phytotherapy of southern Brazil, Paraguay, Uruguay and Argentina belong to this section: e.g. B. trimera, $B$. articulata and B. crispa. Among the remaining species of this section, B. phyteumoides, B. penningtonii. $B$. microcephala and B. gaudichaudiana, were selected to be studied. B. spicata was also analyzed. All these aromatic species are commonly named "carqueja". Although they have different morphological characteristics, when they are commercialized as powders they can not be well identified. The lack of chemical studies on the volatile composition of these species led us to analyze their essential oils in order 
to find a pattern that could be used to discriminate them and to identify any possible adulterations or substitutions. B. gaudichaudiana DC. [BG] is a shrub that grows in Brazil, Paraguay and Argentina, commonly known as "carqueja", "carqueja dulce" or "chilca melosa". It is used in folk medicine as antidiabetic, stomachic, diuretic and antipyretic. Depending on its diterpenoids composition (gaudichaudiosides), it may have different taste properties, from sweet to bitter or neutral. ${ }^{4}$ B. microcephala (Less.) DC. $[\mathrm{BM}]$, also named "carqueija" or "carquejilla", is native to southern Brazil, Paraguay, Uruguay and northeastern Argentina. ${ }^{5}$ The infusion of its aerial parts is used externally for the treatment of rheumatic ailments and to heal wounds. When administered orally, it is hepatic, diuretic, and antidiarrheic. ${ }^{6} B$. penningtonii Heering $[\mathrm{BP}]$ is a shrub native to Uruguay and northern Argentina. ${ }^{5}$ To date, no reports about its chemical composition or local uses have been found. As for B. phyteumoides (Less.) DC. [BY], no ethnobotanical information has been found either. The latter species grows in Argentina, Brazil, Paraguay and Uruguay. B. spicata (Lam.) Baill. [BS] is also known as "chilca blanca" or "pi is í mop", and grows in Brazil, Argentina, Uruguay and Paraguay. ${ }^{1}$ The aerial parts of B. spicata are used in folk medicine as diuretic and digestive. Antioxidant, trypanocidal and antibacterial activities for this species have also been reported..$^{7-9}$

\section{Experimental}

\section{Materials and methods}

Plant materials were collected in different locations of Argentina (Table 1). The botanical identification was done by our group (Dr. M. Gattuso and Dr. S. Gattuso) and voucher specimens of wild materials were deposited in the National University of Rosario (UNR), Argentina.

The oils were obtained from the aerial parts (100 g of air dried material) by hydrodistillation for $3 \mathrm{~h}$ using a Clevenger apparatus. ${ }^{10}$ The collected oil samples were dried over anhydrous sodium sulfate and stored at $2{ }^{\circ} \mathrm{C}$ until analyzed. The analysis of the oils was performed on a Perkin Elmer Clarus 500 GC/FID/MS system, with a special configuration. One injector (split ratio: 1:100) was connected by a flow splitter to two capillary columns: a) one by polyethylene glycol 20,000 and b) other by $5 \%$ phenyl-95\% methyl silicone, both $60 \mathrm{~m} \times 0.25 \mathrm{~mm}$ with $25 \mu \mathrm{m}$ film thickness. The polar column was connected to a FID, whereas the non-polar column was connected to a FID and a quadrupolar mass detector $(70 \mathrm{eV})$, split by a vent system (MS Vent ${ }^{\mathrm{TM}}$ ) and a restrictor, to obtain a split ratio FID/MS of 2:1. Helium was used as mobile phase at a constant flow rate of $1.87 \mathrm{~mL} \mathrm{~min}^{-1}$. The temperature was programmed according to the following gradient: from 90 to $225{ }^{\circ} \mathrm{C}$ at $3{ }^{\circ} \mathrm{C} \mathrm{min}{ }^{-1}$, and then isothermal for $15 \mathrm{~min}$. The injector and both FIDs were set at $255^{\circ} \mathrm{C}$ and $275^{\circ} \mathrm{C}$, respectively. The injection volume was $0.2 \mu \mathrm{L}$ of a $10 \%$ solution of the oil in ethanol. The temperature of the transference line and the ion source were $180{ }^{\circ} \mathrm{C}$ and $150{ }^{\circ} \mathrm{C}$ respectively; the range of masses was $40-300 \mathrm{Da}$, $10 \mathrm{scan} \mathrm{s}^{-1}$. The identification of the compounds was performed by comparison of the retention indices (relative to $\mathrm{C}_{8}-\mathrm{C}_{20} n$-alkanes) obtained in both columns, with those of reference compounds. Additionally, each mass spectra obtained was compared to those from literature libraries and from a laboratory-developed mass spectra library. ${ }^{11,12}$ The percentage composition was determined by using the single area percentage method, without considering corrections for response factors. The lowest response obtained from each column was considered.

\section{Results and Discussion}

The hydrodistillation of the different materials yielded oils in the range from 0.1 to $0.2 \%$. A total of 93 compounds, representing between $74-96 \%$ of each oil, were identified. The percentage composition of each sample is listed in Table 2. The composition of the essential oils analyzed in this study were qualitatively

Table 1. Collection data of analyzed samples of Baccharis spp

\begin{tabular}{lcccc}
\hline Sample & \multicolumn{1}{c}{ Species } & Voucher & Site & Date \\
\hline BG 1 & B. gaudichaudiana & 1564 & Prov. Misiones & $2005-10$ \\
BG 2 & B. gaudichaudiana & 1655 & Prov. Misiones & $2006-02$ \\
BP & B. penningtonii & 1650 & Prov. Entre Ríos & $2006-03$ \\
BY & B. phyteumoides & 1888 & Prov. Santa Fe & $2007-02$ \\
BM & B. microcephala & 1657 & Prov. Misiones & $2006-02$ \\
SP 1 & B. spicata & 1647 & Prov. Santa Fe & $2006-03$ \\
SP 2 & B. spicata & Prov. Corrientes & $2007-03$ \\
\hline
\end{tabular}


but not quantitatively similar. Spathulenol (8.2-38.2\%) and caryophyllene oxide (3.6-22.4\%) were present in all the species in high percentages, except for [BP] (1.9 and $2.9 \%$ respectively). BG 1 and BG 2 displayed a high content of $\beta$-pinene and limonene (25.1-31.0\% and 4.6-7.2\% respectively). BS 1 and BS 2 also showed a high content of the latter substances (7.1-26.0\% and $8.1-4.4 \%$ respectively), with the addition of $\beta$-caryophyllene which was also found in a high percentage (8.3-7.7\%). [BY] was characterized by a high content of $\beta$-selinene and phytol acetate E (12.9 and 16.2\% respectively). The hydrocarbon monoterpene fraction was minoritary in BM, whereas humulene epoxide II and globulol were found in high amounts. Particularly in BP, the major compound identified was germacrene $\mathrm{D}$ with a percentage of about $29.3 \%$.

Table 2. Chemical composition (\%) of the essential oils of Baccharis spp

\begin{tabular}{|c|c|c|c|c|c|c|c|c|}
\hline \multirow[b]{2}{*}{$\mathrm{RI}^{\mathrm{a}}$} & \multirow[b]{2}{*}{ Identification } & \multicolumn{2}{|c|}{ B. gaudichaudiana } & \multirow{2}{*}{$\frac{\text { B. penningtonii }}{\mathrm{BP}}$} & \multirow{2}{*}{$\begin{array}{c}\text { B. phyteumoides } \\
\text { BY }\end{array}$} & \multirow{2}{*}{ B. microcephala } & \multicolumn{2}{|c|}{ B. spicata } \\
\hline & & BG 1 & BG 2 & & & & BS 1 & BS 2 \\
\hline 931 & $\alpha$-thujene & $\mathrm{t}$ & $\mathrm{t}$ & $\mathrm{t}$ & $\mathrm{t}$ & - & - & - \\
\hline 938 & $\alpha$-pinene & 2.2 & 3.2 & 0.5 & $\mathrm{t}$ & $\mathrm{t}$ & 0.5 & 1.9 \\
\hline 951 & $\alpha$-fenchene & $\mathrm{t}$ & $\mathrm{t}$ & - & $\mathrm{t}$ & - & - & - \\
\hline 955 & camphene & $\mathrm{t}$ & $\mathrm{t}$ & $\mathrm{t}$ & - & - & - & $\mathrm{t}$ \\
\hline 960 & thuja-2,4 (10)-diene & $\mathrm{t}$ & $\mathrm{t}$ & - & - & - & - & - \\
\hline 975 & sabinene & 0.4 & $\mathrm{t}$ & 0.8 & 0.7 & - & $\mathrm{t}$ & $\mathrm{t}$ \\
\hline 984 & myrcene & $\mathrm{t}$ & $\mathrm{t}$ & 0.6 & 1.3 & - & $\mathrm{t}$ & $\mathrm{t}$ \\
\hline 985 & $\beta$-pinene & 25.1 & 31.0 & 0.8 & 1.0 & - & 7.1 & 26.0 \\
\hline 1001 & $\delta$-2-carene & $\mathrm{t}$ & - & - & - & - & - & - \\
\hline 1016 & $\alpha$-terpinene & $\mathrm{t}$ & $\mathrm{t}$ & $\mathrm{t}$ & $\mathrm{t}$ & - & $\mathrm{t}$ & $\mathrm{t}$ \\
\hline 1029 & (Z)- $\beta$-ocimene & $\mathrm{t}$ & $\mathrm{t}$ & $\mathrm{t}$ & $\mathrm{t}$ & - & - & - \\
\hline 1030 & $p$-cymene & - & - & 1.1 & 1.6 & $\mathrm{t}$ & - & 0.4 \\
\hline 1031 & limonene & 4.6 & 7.2 & 1.5 & 8.7 & $\mathrm{t}$ & 8.1 & 4.4 \\
\hline 1032 & $\beta$-phellandrene & $\mathrm{t}$ & $\mathrm{t}$ & 0.2 & $\mathrm{t}$ & - & $\mathrm{t}$ & 0.4 \\
\hline 1034 & 1,8-cineole & $\mathrm{t}$ & - & 1.5 & 2.2 & $\mathrm{t}$ & - & - \\
\hline 1049 & (E)- $\beta$-ocimene & $\mathrm{t}$ & $\mathrm{t}$ & $\mathrm{t}$ & $\mathrm{t}$ & - & - & 0.4 \\
\hline 1062 & $\gamma$-terpinene & 0.1 & $\mathrm{t}$ & $\mathrm{t}$ & $\mathrm{t}$ & - & $\mathrm{t}$ & $\mathrm{t}$ \\
\hline 1087 & terpinolene & 0.1 & $\mathrm{t}$ & $\mathrm{t}$ & $\mathrm{t}$ & - & $\mathrm{t}$ & $\mathrm{t}$ \\
\hline 1092 & $\rho$-cimenene & $\mathrm{t}$ & $\mathrm{t}$ & $\mathrm{t}$ & $\mathrm{t}$ & $\mathrm{t}$ & $\mathrm{t}$ & $\mathrm{t}$ \\
\hline 1096 & 6-camphene & $\mathrm{t}$ & $\mathrm{t}$ & - & - & - & - & - \\
\hline 1098 & linalool & - & - & $\mathrm{t}$ & $\mathrm{t}$ & 1.1 & $\mathrm{t}$ & $\mathrm{t}$ \\
\hline 1101 & $n$-nonanal & - & - & $\mathrm{t}$ & - & $\mathrm{t}$ & $\mathrm{t}$ & $\mathrm{t}$ \\
\hline 1110 & $1,3,8-\rho$-menthatriene & $\mathrm{t}$ & - & - & - & - & - & - \\
\hline 1125 & $\alpha$-campholenal & 0.1 & $\mathrm{t}$ & - & - & - & - & - \\
\hline 1138 & trans-pinocarveol & 0.8 & 2.8 & - & - & - & - & $\mathrm{t}$ \\
\hline 1164 & pinocarvone & 0.9 & 1.8 & $\mathrm{t}$ & $\mathrm{t}$ & - & $\mathrm{t}$ & $\mathrm{t}$ \\
\hline 1179 & terpinen-4-ol & $\mathrm{t}$ & 0.4 & $\mathrm{t}$ & $\mathrm{t}$ & $\mathrm{t}$ & - & $\mathrm{t}$ \\
\hline 1188 & $\alpha$-terpineol & $\mathrm{t}$ & 1 & $\mathrm{t}$ & - & $\mathrm{t}$ & - & $\mathrm{t}$ \\
\hline 1195 & myrtenal & 2.5 & 4.3 & $\mathrm{t}$ & - & - & 0.3 & $\mathrm{t}$ \\
\hline 1207 & verbenone & $\mathrm{t}$ & $\mathrm{t}$ & - & - & - & - & - \\
\hline 1215 & trans-carveol & $\mathrm{t}$ & $\mathrm{t}$ & - & - & - & - & - \\
\hline 1217 & $\beta$-cyclocitral & $\mathrm{t}$ & $\mathrm{t}$ & $\mathrm{t}$ & $\mathrm{t}$ & - & - & $\mathrm{t}$ \\
\hline 1244 & carvone & 0.1 & 0.4 & $\mathrm{t}$ & $\mathrm{t}$ & - & $\mathrm{t}$ & $\mathrm{t}$ \\
\hline 1267 & geranial & $\mathrm{t}$ & $\mathrm{t}$ & - & - & - & - & - \\
\hline 1272 & perilla aldehyde & $\mathrm{t}$ & $\mathrm{t}$ & - & - & - & - & - \\
\hline 1275 & $\rho$-menth-1-en-7-al & $\mathrm{t}$ & $\mathrm{t}$ & - & - & - & - & - \\
\hline 1295 & perilla alcohol & $\mathrm{t}$ & $\mathrm{t}$ & - & - & - & - & - \\
\hline 1320 & 2E-,4E-decadienal & $\mathrm{t}$ & $\mathrm{t}$ & $\mathrm{t}$ & - & - & - & - \\
\hline 1326 & myrtenyl acetate & $\mathrm{t}$ & $\mathrm{t}$ & - & - & - & - & - \\
\hline 1349 & $\alpha$-terpinyl acetate & $\mathrm{t}$ & $\mathrm{t}$ & $\mathrm{t}$ & $\mathrm{t}$ & - & - & $\mathrm{t}$ \\
\hline 1350 & $\alpha$-cubebene & $\mathrm{t}$ & $\mathrm{t}$ & $\mathrm{t}$ & $\mathrm{t}$ & - & - & $\mathrm{t}$ \\
\hline
\end{tabular}


Table 2. continuation

\begin{tabular}{|c|c|c|c|c|c|c|c|c|}
\hline \multirow[b]{2}{*}{$\mathrm{RI}^{\mathrm{a}}$} & \multirow[b]{2}{*}{ Identification } & \multicolumn{2}{|c|}{ B. gaudichaudiana } & \multirow{2}{*}{$\begin{array}{c}\text { B. penningtonii } \\
\mathrm{BP} \\
\end{array}$} & \multirow{2}{*}{$\begin{array}{c}\text { B. phyteumoides } \\
\text { BY }\end{array}$} & \multirow{2}{*}{$\begin{array}{c}\text { B. microcephala } \\
\text { BM }\end{array}$} & \multicolumn{2}{|c|}{ B. spicata } \\
\hline & & BG 1 & BG 2 & & & & BS 1 & BS 2 \\
\hline 1372 & $\alpha$-ylangene & - & - & $\mathrm{t}$ & $\mathrm{t}$ & $\mathrm{t}$ & - & - \\
\hline 1376 & isoledene & $\mathrm{t}$ & - & - & - & - & $\mathrm{t}$ & $\mathrm{t}$ \\
\hline 1379 & $\alpha$-copaene & 0.2 & $\mathrm{t}$ & $\mathrm{t}$ & $\mathrm{t}$ & $\mathrm{t}$ & 1.0 & 0.5 \\
\hline 1388 & $\beta$-bourbonene $+\beta$-elemene & 0.4 & $\mathrm{t}$ & 2.3 & 8.5 & $\mathrm{t}$ & 1.0 & 1.2 \\
\hline 1403 & methyl eugenol & $\mathrm{t}$ & - & - & - & - & - & $\mathrm{t}$ \\
\hline 1408 & $\alpha$-gurjunene & - & - & - & - & $\mathrm{t}$ & - & - \\
\hline 1409 & longifolene & - & - & $\mathrm{t}$ & - & - & $\mathrm{t}$ & $\mathrm{t}$ \\
\hline 1418 & $\beta$-caryophyllene & 1.3 & 1.4 & 4.9 & 2.3 & 0.9 & 8.3 & 7.7 \\
\hline 1432 & $\beta$-copaene & 0.2 & $\mathrm{t}$ & 0.4 & $\mathrm{t}$ & $\mathrm{t}$ & - & $\mathrm{t}$ \\
\hline 1436 & neryl acetone & $\mathrm{t}$ & $\mathrm{t}$ & $\mathrm{t}$ & $\mathrm{t}$ & $\mathrm{t}$ & - & $\mathrm{t}$ \\
\hline 1443 & aromadendrene & 0.1 & $\mathrm{t}$ & $\mathrm{t}$ & $\mathrm{t}$ & 1.6 & $\mathrm{t}$ & 0.3 \\
\hline 1450 & cis-muurola-3,5-diene & $\mathrm{t}$ & $\mathrm{t}$ & $\mathrm{t}$ & - & - & - & $\mathrm{t}$ \\
\hline 1458 & $\alpha$-humulene & 0.2 & 1.2 & 0.7 & 1.8 & 1 & 2.8 & 2.5 \\
\hline 1461 & alloaromadendrene & 0.1 & - & 0.9 & $\mathrm{t}$ & $\mathrm{t}$ & 0.5 & 0.4 \\
\hline 1474 & trans-cadina-1(6),4 diene & $\mathrm{t}$ & $\mathrm{t}$ & $\mathrm{t}$ & $\mathrm{t}$ & $\mathrm{t}$ & $\mathrm{t}$ & $\mathrm{t}$ \\
\hline 1482 & $\gamma$-muurolene & 0.3 & $\mathrm{t}$ & 1.2 & $\mathrm{t}$ & $\mathrm{t}$ & 0.6 & 0.6 \\
\hline 1485 & $\alpha$-amorphene & - & - & 0.9 & 3.7 & - & $\mathrm{t}$ & $\mathrm{t}$ \\
\hline 1486 & germacrene D & 0.1 & 0.4 & 29.3 & 2.1 & - & 3.1 & 6.3 \\
\hline 1490 & calamenene 1,11-epoxide & - & - & - & - & $\mathrm{t}$ & 0.3 & $\mathrm{t}$ \\
\hline 1490 & $\beta$-selinene & - & - & 0.9 & 12.9 & - & $\mathrm{t}$ & 0.5 \\
\hline 1492 & trans-muurola-4(14), 5-diene & - & - & $\mathrm{t}$ & $\mathrm{t}$ & - & 2.9 & $\mathrm{t}$ \\
\hline 1500 & bicyclogermacrene $+\alpha$-muurolene & 0.9 & 0.6 & 3.8 & 2.4 & $\mathrm{t}$ & 1.1 & 2.8 \\
\hline 1512 & $\gamma$-cadinene & - & $\mathrm{t}$ & 0.8 & $\mathrm{t}$ & $\mathrm{t}$ & $\mathrm{t}$ & 0.6 \\
\hline 1513 & $\delta$-amorphene & 1.1 & $\mathrm{t}$ & $\mathrm{t}$ & $\mathrm{t}$ & 0.6 & 2.3 & 2.5 \\
\hline 1531 & trans-calamenene & $\mathrm{t}$ & $\mathrm{t}$ & $\mathrm{t}$ & $\mathrm{t}$ & $\mathrm{t}$ & 0.3 & 0.2 \\
\hline 1535 & trans-cadina-1,4-diene & $\mathrm{t}$ & - & $\mathrm{t}$ & $\mathrm{t}$ & $\mathrm{t}$ & $\mathrm{t}$ & $\mathrm{t}$ \\
\hline 1540 & $\alpha$-cadinene & 0.2 & $\mathrm{t}$ & $\mathrm{t}$ & $\mathrm{t}$ & - & $\mathrm{t}$ & $\mathrm{t}$ \\
\hline 1545 & $\alpha$-calacorene & 0.3 & $\mathrm{t}$ & 1 & $\mathrm{t}$ & 1.1 & 0.9 & 0.6 \\
\hline 1563 & E-nerolidol & - & - & 1 & 1.6 & 2.6 & $\mathrm{t}$ & $\mathrm{t}$ \\
\hline 1567 & $\beta$-calacorene & $\mathrm{t}$ & $\mathrm{t}$ & $\mathrm{t}$ & $\mathrm{t}$ & $\mathrm{t}$ & $\mathrm{t}$ & $\mathrm{t}$ \\
\hline 1579 & spathulenol & 38.2 & 22.0 & 1.9 & 8.2 & 17.4 & 18.7 & 13.1 \\
\hline 1582 & caryophyllene oxide & 8.7 & 14.0 & 2.9 & 5.7 & 22.4 & 15.1 & 3.6 \\
\hline 1591 & globulol & - & - & - & - & 10.1 & - & $\mathrm{t}$ \\
\hline 1592 & epiglobulol (viridiflorol) & - & $\mathrm{t}$ & $\mathrm{t}$ & - & 0.6 & $\mathrm{t}$ & $\mathrm{t}$ \\
\hline 1594 & salvial-4(14)-en-1-one & 0.1 & 1.4 & - & $\mathrm{t}$ & - & $\mathrm{t}$ & - \\
\hline 1609 & humulene epoxide II & 0.5 & 3.2 & - & 1.1 & 12.4 & 2.8 & - \\
\hline 1627 & cubenol-1-epi & 0.2 & $\mathrm{t}$ & 1.5 & $\mathrm{t}$ & 0.8 & 0.7 & 1.2 \\
\hline 1640 & caryophylla-4(12), 8 (13)-dien-5-ol & - & $\mathrm{t}$ & - & $\mathrm{t}$ & 1.2 & $\mathrm{t}$ & $\mathrm{t}$ \\
\hline 1641 & $\alpha$-epi-cadinol & - & - & 0.7 & 0.8 & $\mathrm{t}$ & $\mathrm{t}$ & 1.8 \\
\hline 1642 & $\alpha$-epi-muurolol & 0.4 & $\mathrm{t}$ & 1.1 & $\mathrm{t}$ & $\mathrm{t}$ & 1.1 & 0.7 \\
\hline 1645 & $\alpha$-muurolol & - & - & 1.2 & $\mathrm{t}$ & $\mathrm{t}$ & 0.5 & 0.5 \\
\hline 1653 & $\alpha$-cadinol & 0.3 & $\mathrm{t}$ & 2.0 & $\mathrm{t}$ & $\mathrm{t}$ & 0.7 & 1.0 \\
\hline 1675 & caladene & - & - & $\mathrm{t}$ & $\mathrm{t}$ & 3.5 & $\mathrm{t}$ & $\mathrm{t}$ \\
\hline 1672 & caryophyllene 14-hydroxy-9-epi-(E) & $\mathrm{t}$ & $\mathrm{t}$ & $\mathrm{t}$ & $\mathrm{t}$ & $\mathrm{t}$ & 0.6 & 0.2 \\
\hline 1685 & germacra-4(15),5,10(14)-trien-1 $\alpha$-ol ${ }^{\text {b }}$ & - & - & 2.5 & $\mathrm{t}$ & $\mathrm{t}$ & $\mathrm{t}$ & 3.0 \\
\hline 1686 & eudesma-4(15),7-dien-1 $\beta$-ol (impure) & - & - & 0.9 & $\mathrm{t}$ & - & $\mathrm{t}$ & $\mathrm{t}$ \\
\hline 1693 & cuprenen-1-ol (4-) & - & - & - & - & $\mathrm{t}$ & - & - \\
\hline 1717 & curcuphenol & - & - & 0.9 & - & - & - & - \\
\hline 1769 & $\gamma$-curcumen-15-al & 0.3 & $\mathrm{t}$ & - & - & - & 0.4 & 0.8 \\
\hline \multirow[t]{2}{*}{2217} & phytol acetate E & 0.6 & $\mathrm{t}$ & 3.6 & 16.2 & $\mathrm{t}$ & 3.0 & 3.0 \\
\hline & $\%$ total & 91.6 & 96.3 & 74.3 & 82.8 & 77.2 & 84.5 & 89.0 \\
\hline
\end{tabular}

Compounds are listed in order of elution on non-polar column. t: traces $(\leq 0.1 \%)$; ${ }^{\mathrm{a} E x p e r i m e n t a l ~ R e t e n t i o n ~ I n d e x ~(R I) ~ o n ~ n o n-p o l a r ~ c o l u m n . ~}{ }^{\mathrm{b}} \mathrm{Correct}$ isomer not identified. 
Regarding the industrial uses, two groups of Baccharis species with economic implications in South America can be defined: those named "carqueja" with widespread ethnomedicinal, phytotherapeutic and food uses, and $B$. dracunculifolia, named "vassoura", largely employed in the fragrance industry due to the high content of E-nerolidol in its essential oil. Other Baccharis oils have been previously described, some of them also showing a predominant content of this alcoholic sesquiterpene (B. articulata, $B$. cordobensis, B. crispa, B. rufescens). ${ }^{13-16}$ Two species, $B$. crispa and B. articulata, from section Caulopterae, are both rich in E-nerolidol and spathulenol.

Four species belonging to section Caulopterae, and Baccharis spicata, all of them known as "carqueja", were analyzed herein. The composition of these essential oils showed the same qualitative pattern, but presented quantitative differences. Likewise in other species of this mentioned section, spathulenol or germacrene D (biosynthetic precursor of the former compound) were found as the main constituents, in agreement with other species of this section. The chemical composition of the essential oil of Baccharis microcephala from Brazil reported by SimoesPires et al. ${ }^{16}$ showed a similar pattern but quantitatively different from our results, with predominance of $\alpha$-cadinol, spathulenol, caryophyllene oxide and globulol. These quantitative discrepancies can be explained by differences in the response to geographic, climatic, phenological or edaphic patterns. The qualitative pattern similarities within the studied species were expected as they belong to a common genus and four of them to the same section.

B. trimera is the most common and studied species among the so-called "carquejas". For this reason it was included in the latest edition of the Brazilian Pharmacopoeia ${ }^{17}$ and was also proposed to be included in the next edition of the Argentine Pharmacopoeia. ${ }^{18}$ Carquejyl acetate and carquejol, which are present in its essential oil, were proposed as unequivocal chemotaxonomic marker of this species. ${ }^{16}$ Nevertheless, some populations of $B$. trimera lacking these compounds, have been recently described suggesting the existence of a novel chemotype or chemical variations that arise as a result of different environmental conditions. ${ }^{19,20}$ This feature emphasizes the necessity for an accurate identification of the pharmacopoeial quality of carqueja. In Brazil, the magnitude of the populations of B. trimera assures its sustainability. ${ }^{21}$ However, in Argentina this species is under threat due to its great demand in the local traditional medicine, phytotherapy and food industry. ${ }^{22}$ As a result, many adulterations with other Baccharis spp. have been detected in our country. For this reason, the essential oils of other species of Baccharis belonging to the section Caulopterae and growing in the same habitat were analyzed herein. B. spicata was also included as it is another common adulterant of carqueja. The composition of the oils of these species was studied in order to assess whether they contain the chemotaxonomic markers that have been proposed for B. trimera. Finally, we demonstrated that these oxygenated terpenes were not present in any of the analyzed species. These results allow us to ensure that these chemical markers could be the most suitable for the identification of the legitimate carqueja.

\section{Acknowledgements}

This work was supported in part by Projects PICTR-0284, UBA-CYT B019 and B014.

\section{References}

1. Zardini, E. M.; Acta Farm. Bonaerense 1984, 3, 77.

2. Giuliano, D. A.; Darwiniana 2001, 39, 131.

3. Ariza Espinar, L.; Bol. Acad. Nac. Cienc. (Córdoba) 1973, 50, 175.

4. Fullas, F.; Hussain, R. A.; Chai, H. B; Pezzuto, J. M.; Soejarto, D. D.; Kinghorn, A. D.; J. Nat. Prod. 1994, 57, 801.

5. Giuliano, D.A. In Flora Fanerogámica Argentina; A. T. Hunziker, ed.; Conicet: Córdoba, 2000, fasc. 66.

6. Pensiero, J.; Muñoz, J.; Martinez, V.; In Alternativas de Sustentabilidad del Bosque Nativo del Espinal. hppt:// www.ambiente.gov.ar/archivos/web/PBVyAP/File/A3/ PIARFON\%20MyE/Etnobotanica.pdf, accessed in May 2008.

7. De Oliveira, S. Q. de; Dal-Pizzol, F.; Moreira, J. C. F.; Schenkel, E. P.; Gosmann, G.; Acta Farm. Bonaerense 2004, 23, 365.

8. Sülsen, V.; Güida, C.; Coussio, J.; Paveto, C.; Muschietti, L.; Martino, V.; Parasitol. Res. 2006, 98, 370.

9. De Oliveira, S. Q. de; Trentin, V. H.; Kappel, V. D.; Barelli, C.; Gosmann, G.; Reginatto, F. H.; Pharm. Biol. 2005, 43, 434.

10. European Pharmacopoeia, vol. 1, Council of Europe: Strasbourg, France, 2005.

11. Adams, R. P.; Identification of Essential Oils Components by Gas Chromatography/Quadrupole Mass Spectroscopy, Allured Publ. Corp.: Carol Stream, USA, 2007.

12. Mc Lafferty, F. W.; Stauffer D. B.; The Wiley/NBS Registry of Mass Spectral Data, Wiley: New York, 2005.

13. Zunino, M. P.; Newton, M. N.; Maestri, D. M.; Zygadlo, J. A.; Planta Med. 1998, 64, 86.

14. Zunino, M. P.; Lopez, M. L.; Faillaci, S. M.; Lopez, A. G.; Ariza Espinar, L.; Zygadlo, J. A.; Flavour Fragr. J. 2000, 15, 151.

15. Zunino, M. P.; López, M. L.; Zydaglo, J. A.; López, A. G.; J. Essent. Oil Res. 2004, 16, 29.

16. Simoes-Pires, C. A.; Debenedetti, S.; Spegazzini, E.; Mentz, L. A.; Matzenbacher, N. I.; Limberger, R. P.; Henriques, A. T.; Pl. Syst. Evol. 2005, 253, 23. 
17. Farmacopeia Brasileira, 4a. ed., vol.1, Ed. Ateneu: Rio de Janeiro, Brasil, 2003

18. Ferraro, G., Coordinadora; Subcomisión de Medicamentos Fitoterápicos de la Farmacopea Argentina, 2008, personal communication.

19. Silva, F. G.; Oliveira, C. B. A.; Pinto, J. E. B. P.; Nascimento,V. E.; Santos, S. C.; Seraphind, J. C.; Ferri, P. H.; J. Braz. Chem. Soc. 2007, 18, 990.

20. Lago, J. H. G.; Romoff, P.; Fávero, O. A.; Souza, F. O.; Soares, M. G.; Baraldi, P. T.; Correa, A. G.; Biochem. Syst. Ecol. 2008, 36,737 .
21. Steenbock, W.; In Search of Sustainable Management of Carqueja (Baccharis trimera Lers) in the Central Region of Paraná, Southern Brazil; Alexiades, M.N. y Shanley, P. eds.; Productos Forestales, Medios de Subsistencia y Conservación. Estudios de Caso sobre Sistemas de Manejo de Productos Forestales No Maderables. Vol 3 - América Latina. CIFOR (Centro para la Investigación Forestal Internacional): Bogor, Indonesia, 2004.

22. Martínez, G. J.; Acta Farm. Bonaerense 2005, 24, 575.

Received: July 16, 2008

Web Release Date: July 31, 2009 